\title{
五轴线性刀路的转接光顺及轨迹生成算法
}

\author{
赵 欢 张永红 丁 汉 \\ (华中科技大学数字制造装备与技术国家重点实验室 武汉 430074)
}

\begin{abstract}
摘要: 线性刀路是五轴加工中广泛采用的刀具路径形式。由于线性刀路的切向和曲率均不连续, 容易引起机床振动, 降低加 工质量和加工效率。已有的方法仍然存在一些问题, 同步参数化、误差控制以及实时性无法同时满足, 在规划进给速度时没 有考虑五轴机床的非线性运动学特性。为此，提出了一种新的路径光顺方法。该方法采用 2 条 3 次 Bézier 曲线对线性刀路进 行转接光顺, 提高几何连续性: 一条 Bézier 曲线对刀尖点位置进行光顺; 为了保证刀轴矢量单位化, 用 2 个欧拉角表示刀轴 矢量, 从球面坐标系映射到平面笛卡尔坐标系, 用另一条 Bézier 曲线对其光顺。接着, 给出了基于转接光顺的轨迹生成算法。 该算法考虑非线性运动学特性, 结合前瞻技术和 7 段式 $S$ 型速度规划方法, 实时规划出满足伺服约束的平滑进给速度。仿真 与试验结果表明: 所提出的刀路光顺与轨迹生成算法能够规划出加速度连续的进给速度曲线, 提高进给速度, 生成的轨迹满 足驱动约束, 适用于实时环境。
\end{abstract}

关键词: 转接光顺; 轨迹生成; 5 轴; Bézier

中图分类号: TP273

\section{A Corner Rounding and Trajectory Generation Algorithm for Five-axis Linear Toolpath}

\author{
ZHAO Huan ZHANG Yonghong DING Han
}

(State Key Laboratory of Digital Manufacturing Equipment and Technology, Huazhong University of Science and Technology, Wuhan 430074)

\begin{abstract}
Linear tool paths are still the most widely used format of tool path in five-axis machining. Tangential and curvature discontinuities of linear tool path may lead to vibration of the machine, which inevitably result in poor machining efficiency and quality. To solve this issue, different smoothing methods is developed in the existing literatures. There exist some problems such as parameter synchronization, error restriction and real-time that cannot be realized simultaneously, the nonlinear kinematics are not taken into account while feedrate scheduling. A novel local path smoothing for five-axis machine is proposed. Segment junctions of the linear tool path in the workpiece coordinate system are blended by two transition cubic- Bézier curves. One cubic Bézier curve is used to smooth the segment junction of tool tip position path. To ensure magnitude of the orientation vector always results in unit magnitude, it is represented by two Eular angles and mapped form spherical coordinates to Cartesian plane, where another Bézier curve is used. Then, a trajectory generation algorithm for local path smoothing is proposed. The proposed trajectory generation algorithm takes the nonlinear kinematics into account, and look-ahead and seven segment S-shape feedrate scheduling method is employed to schedule a feedrate profile while respecting drive constrains. Finally, the experiments are conducted to verify the feasibility and efficiency of the proposed algorithm.
\end{abstract}

Key words: corner rounding; trajectory generation; five-axis; Bézier

\section{0 前言}

线性刀具路径仍然是目前工业应用最广泛的 数控代码格式 ${ }^{[1]}$ 。在线性路径的连接点处, 刀具的

* 国家自然科学基金(51405175, 51535004 和 51323009)和国家科技重大专 项(2012ZX04001-012-01-05)资助项目。20170116 收到初稿, 20170522 收到修改稿
切向和曲率均不连续, 这种不连续往往导致进给 速度波动, 在实际加工过程中容易引起机床振动, 严重降低加工效率和表面质量。为了克服小线段 带来的问题, 可以对小线段路径进行光顺处理, 提高路径的连续性, 从而提高加工效率, 并改善 加工质量 ${ }^{[2]}$ 。

针对 3 轴数控加工, 通过对刀路进行光顺来提 高加工性能, 已有较为系统的研究成果 ${ }^{[3-5]}$ 。然而, 
对于五轴加工, 目前仍处在探索的阶段。目前五轴 刀路光顺方法主要有两种: 拟合光顺, 转接光顺。 拟合光顺采用高阶样条曲线来对离散小线段进行 逼近或者插值拟合 ${ }^{[4-7]}$ 。拟合光顺算法难以控制拟 合精度, 且计算量比较大, 很难在实时计算环境中 使用 ${ }^{[8-9]}$ 。

转接光顺的思路是在小线段连接点用圆弧或高 阶连续的样条曲线过渡。文献[10-11]用 2 条曲线分 别对刀尖点和刀轴上一点进行转接光顺, 每条曲线 的阶数相同, 刀尖点和刀轴上一点的轨迹都满足 G2 连续, 但是没有实现同步参数化, 各伺服轴的速度 和加速度在转接点处并不连续。同时这种耦合的光 顺方法不能保证刀轴方向矢量为单位矢量。 TULSYAN 等 ${ }^{[12]}$ 对刀尖点和刀轴矢量分别进行转 接, 但是该光顺方法亦不能保证刀轴矢量单位化, 且需要用雅可比迭代求解多元非线性方程组, 采用 的速度规划方法也存在迭代过程, 从路径光顺到轨 迹插补计算需要耗费大量时间。BI 等 ${ }^{[13]}$ 在机床坐标 系对平动轴和转动轴分别用 3 次 Bézier 曲线进行光 顺, 并进行轨迹插补计算。由于旋转轴的旋转运动, 刀尖点轨迹是一条偏离理论轨迹的空间曲线, 降低 了加工精度, 且旋转轴和平动轴是不同的传动方式, 速度规划时并不适合将二者统一考虑。

五轴加工需要同步考虑刀具相对于工件的位 置和方向, 因此较传统的 2 轴或者 3 轴加工, 其路 径光顺及插补计算更加复杂, 主要存在以下难题: 刀尖点轨迹和刀轴方向的同步参数化与误差控制; 从工件坐标系到机床坐标系是非线性映射, 各伺服 轴的运动不仅与刀具的运动有关, 同时也受非线性 映射的影响; 另外考虑到实际应用, 还需具备实时 性。目前这些问题没有得到有效解决, 仍然是研究 的热点。

围绕这些难题, 本文提出了一种新的的刀路光 顺及轨迹生成算法。首先采用 2 条 Bézier 曲线对前 瞻窗口内的刀具路径进行光顺, 一条对刀尖点的轨 迹进行光顺, 另一条对刀轴矢量光顺。为了保证光 顺之后的刀轴矢量仍然是单位矢量, 将刀轴矢量用 2 个欧拉角表示, 从球面坐标系映射到平面笛卡尔 坐标系。接着给出了基于转接光顺的轨迹生成算法。 该算法考虑机床特性与运动学约束, 结合前瞻技术 与 7 段式 $\mathrm{S}$ 型速度规划方法, 实时规划出跃度有限 的进给速度曲线。各伺服轴的速度与加速度光滑连 续, 满足最大值约束。

论文的主要内容安排如下: 第 1 节介绍 G2 连 续的转接光顺方法; 第 2 节介绍转接光顺的轨迹生 成; 第 3 节是仿真与试验; 第 4 节是结论。

\section{$1 \mathrm{G} 2$ 连续转接光顺}

路径的 G2 连续是提高进给率、减小机床振动 的前提。 3 次曲线是实现 $\mathrm{G} 2$ 连续性的最低阶次曲 线, 本文采用 3 次 Bézier 曲线对五轴线性路径的刀 尖点和刀轴矢量分别进行转接光顺, 实现刀具平移 运动和旋转运动的 G2 连续, 并保证二者的参数化 同步。

3 次 Bézier 曲线的定义如下 ${ }^{[14]}$

$$
\boldsymbol{B}(t)=\sum_{j=0}^{3} \boldsymbol{B}_{j}(1-t)^{3-j} t^{j}
$$

式中, $\boldsymbol{B}_{j}$ 是对应的 Bézier 曲线的控制点; $t$ 是 Bézier 曲线的参数, $0 \leqslant t \leqslant 1$ 。

\section{1 刀尖点的光顺}

如图 1 所示, 两条相邻线性路径 $\boldsymbol{P}_{0} \boldsymbol{P}_{1}$ 和 $\boldsymbol{P}_{1} \boldsymbol{P}_{2}$ 由 点 $\boldsymbol{P}_{0} 、 \boldsymbol{P}_{1}$ 和 $\boldsymbol{P}_{2}$ 定义, $\boldsymbol{T}_{1}$ 是线段 $\boldsymbol{P}_{0} \boldsymbol{P}_{1}$ 的单位矢量, $\boldsymbol{T}_{2}$ 是线段 $\boldsymbol{P}_{1} \boldsymbol{P}_{2}$ 的单位矢量。过渡曲线的 4 个控制点为: $\left\{\boldsymbol{B}_{0}, \boldsymbol{B}_{1}, \boldsymbol{B}_{2}, \boldsymbol{B}_{3}\right\}$, 且

$$
d=\left\|\boldsymbol{B}_{0} \boldsymbol{P}_{1}\right\|=\left\|\boldsymbol{P}_{1} \boldsymbol{B}_{3}\right\|
$$

为转接长度。基于上述构造方式, 将式(2)代入 式(1)得到简化的 Bézier 曲线的表达式

$$
\boldsymbol{B}_{\boldsymbol{P}}(t)=\boldsymbol{P}_{1}-d(1-t)^{3} \boldsymbol{T}_{1}+d t^{3} \boldsymbol{T}_{2}
$$

曲线与原线性路径的最大距离出现在 $t=0.5$ 处, 且最大偏差为

$$
\varepsilon=\frac{d}{4} \sin \frac{\theta}{2}
$$

因此, 当给定刀尖点的光顺误差 $\varepsilon$, 转接长度 $d$ 可以如下计算

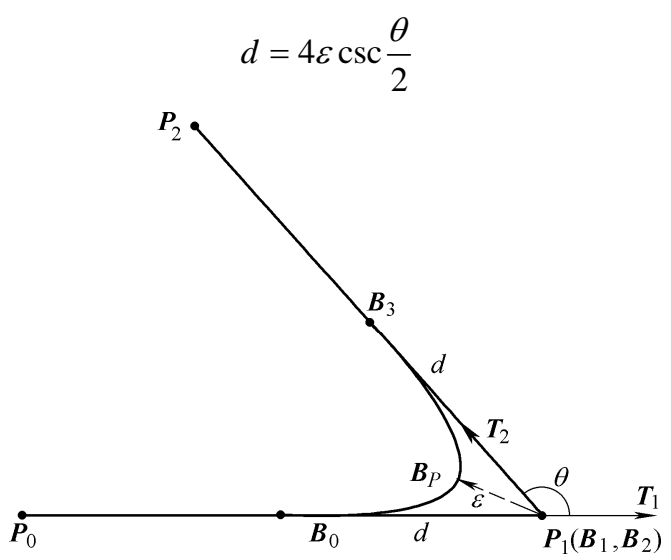

图 1 相邻线性路径的 Bézier 曲线光顺

\section{2 刀轴方向的光顺}

刀轴方向 $\boldsymbol{o}=\left[o_{i}, o_{j}, o_{k}\right]$ 也同样采用 3 次 Bézier 曲 线进行光顺。但是与刀尖点的光顺不同的是, 为了 保证刀轴矢量是单位矢量, 将刀轴方向用 2 个欧拉 
角表示

$$
\begin{aligned}
& \beta=\arccos \left(o_{k}\right) \\
& \alpha=\arctan \left(o_{j} / o_{i}\right)
\end{aligned}
$$

刀轴矢量从球面坐标系映射到平面笛卡尔坐标 系, $\boldsymbol{Q}=[\alpha, \beta]$ 表示该平面内与 $\boldsymbol{o}$ 对应的点。当给定 该平面坐标系的转接误差 $\varepsilon_{q}$, 结合式(5)和式(3)即可 构造出刀轴方向的光顺曲线。基于式(6)的映射关 系, $\varepsilon_{q}$ 与刀轴的方向误差 $\varepsilon_{o}$ 存在如下关系。

设 $\boldsymbol{Q}=[\alpha, \beta]$ 对应刀轴方向 $\boldsymbol{o}$; 当 $\alpha 、 \beta$ 分别有一 个微小的增量 $\Delta \alpha 、 \Delta \beta$ 时, 得到的新的点 $\boldsymbol{Q}^{\prime}$ 和刀轴 方向 $\boldsymbol{o}^{\prime}$, 如图 2 所示。设 $\boldsymbol{o}$ 和 $\boldsymbol{o}^{\prime}$ 之间的夹角为 $\varepsilon$, 欧 拉平面坐标系的误差为 $\varepsilon_{\boldsymbol{Q}}=\left\|\boldsymbol{Q}-\boldsymbol{Q}^{\prime}\right\|=\sqrt{\Delta \alpha^{2}+\Delta \beta^{2}}$, 若 $\boldsymbol{o}$ 和 $\boldsymbol{o}$ 之间的最大角度误差为 $\varepsilon_{o}$, 当 $\varepsilon_{q}<\varepsilon_{o}$ 时, 可得到

$$
\varepsilon<\varepsilon_{o}
$$
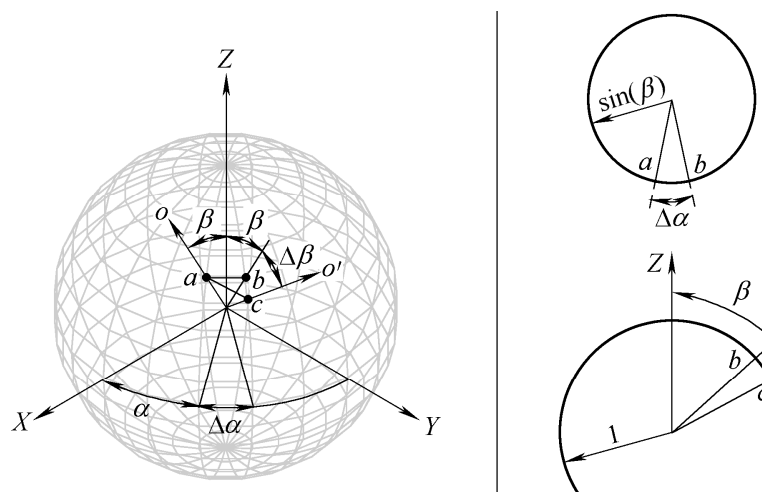

图 2 方向误差的映射关系

下面给出式(7)的证明。

证明: 刀轴矢量与欧拉角的关系体现在 $\Delta a b c$ 中

$$
\left\{\begin{array}{l}
a b=\sin (\beta) \times 2 \sin (\Delta \alpha) \\
b c=2 \sin (0.5 \Delta \beta) \\
a c=2 \sin (0.5 \Delta \varepsilon)
\end{array}\right.
$$

根据三角形边长的关系

$$
|a b|^{2}+|b c|^{2}-2|a b| \cdot|b c| \cos \angle a b c=|a c|^{2}
$$

$a b$ 与 $b c$ 的夹角

$\angle a b c=\arccos (\cos (90-0.5 \Delta \alpha) \cos (180-\beta-0.5 \Delta \beta))$

$\Delta \alpha 、 \Delta \beta$ 和 $\varepsilon$ 非常小 $\left(<1^{\circ}\right)$, 式(8)可简化为

$$
\left\{\begin{array}{l}
a b=(\sin \beta) \times \Delta \alpha \\
b c=\Delta \beta \\
a c=\varepsilon
\end{array}\right.
$$

$\cos (90-\Delta \alpha / 2) \approx 0$, 代入式(10), 可得

$$
\angle a b c \approx 90
$$

将式(11)、式(12)代入式(9)

$$
(\sin \beta)^{2} \Delta \alpha^{2}+\Delta \beta^{2}=\varepsilon^{2}
$$

根据条件 $\varepsilon_{q}<\varepsilon_{o}$, 所以

$$
\varepsilon^{2}<\Delta \alpha^{2}+\Delta \beta^{2}=\varepsilon_{q}^{2}<\varepsilon_{o}^{2}
$$

整理即可得到式(7)。

因此, $\varepsilon_{q}$ 的极值是 $\varepsilon_{o}$ 。不失一般性, 令二者相 等, $\varepsilon_{q}=\varepsilon_{o}$ 。

由于刀具的旋转运动是用欧拉角进行光顺的, 因此需要将欧拉角重新表示成刀轴矢量。与 $\boldsymbol{Q}$ 对应 的刀轴 $\boldsymbol{o}=\left[o_{i}, o_{j}, o_{k}\right]$ 满足

$$
\left\{\begin{array}{l}
o_{i}=\sin \beta \cos \alpha \\
o_{j}=\sin \beta \sin \alpha \\
o_{k}=\cos \beta
\end{array}\right.
$$

\section{3 同步性}

刀尖点位置和刀轴方向的同步性是五轴路径光 顺的另一个重点问题。对于转接光顺而言，同步性 就是要保证同时进入转接和同时结束转接, 且转接 点各伺服轴的速度与加速度均保持连续。

转接之后的路径可分为曲线过渡区域和非过渡 区域。顾名思义，曲线过渡区域就是由 Bézier 曲线 拼接的区域。非转接过渡区域是指转接之后刀具路 径与转接之前的线性路径相重合的路径, 由小线段 路径组成。

相比于用多项式将曲线参数关联到弧长的非线 性同步 ${ }^{[4]}$, 采用同曲线参数进行同步是最可行的方 法, 避免多项式拟合带来的误差, 同时计算量小, 可用于实时的环境。

当转接之后的路径采用同曲线参数进行同步 时，转接长度必须满足以下条件

$$
\begin{aligned}
& \frac{d_{q 1}}{\left\|\boldsymbol{Q}_{i}-\boldsymbol{Q}_{i-1}\right\|}=\frac{d_{p 1}}{\left\|\boldsymbol{P}_{i}-\boldsymbol{P}_{i-1}\right\|}=m_{1} \\
& \frac{d_{q 2}}{\left\|\boldsymbol{Q}_{i+1}-\boldsymbol{Q}_{i}\right\|}=\frac{d_{p 2}}{\left\|\boldsymbol{P}_{i+1}-\boldsymbol{P}_{i}\right\|}=m_{2}
\end{aligned}
$$

式中, $d_{p 1}$ 是 $\boldsymbol{B}_{p}(t)$ 在线性路径上 $\boldsymbol{P}_{i-1} \boldsymbol{P}_{i}$ 的转接长度; $d_{q 1}$ 是 $\boldsymbol{B}_{\boldsymbol{Q}}(t)$ 在线性路径 $\boldsymbol{Q}_{i-1} \boldsymbol{Q}_{i}$ 上的转接长度; $d_{p 2}$ 是 $\boldsymbol{B}_{p}(t)$ 在线性路径 $\boldsymbol{P}_{i} \boldsymbol{P}_{i+1}$ 上的转接长度; $d_{q 2}$ 是 $\boldsymbol{B}_{\boldsymbol{Q}}(t)$ 在路径 $\boldsymbol{Q}_{i} \boldsymbol{Q}_{i+1}$ 上的转接长度, 如图 3 所示。

以下结合图 3 给出式(16)的证明。

证明: 设 $C_{P}(s)$ 表示刀尖点的轨迹曲线, $C_{Q}(s)$ 表示刀轴姿态的轨迹曲线, $s$ 表示刀具路径长度。 $C_{P}(s) 、 C_{Q}(s)$ 在关于 $s$ 的一阶和二阶导数的连续性 直接影响运动的平稳性。很明显, $C_{P}(s) 、 C_{Q}(s)$ 关 于 $s$ 的一阶和二阶导数在非转接区和曲线过渡区域 均连续, 仅需验证在转接点 $\left(\boldsymbol{B}_{0, P}, \boldsymbol{B}_{0, Q}\right)$ 和 $\left(\boldsymbol{B}_{3, P}, \boldsymbol{B}_{3, Q}\right)$ 
处的连续性。首先证明 $C_{P}(s) 、 C_{Q}(s)$ 关于 $s$ 的导数 在转接点 $\left(\boldsymbol{B}_{0, \boldsymbol{P}}, \boldsymbol{B}_{0, \boldsymbol{Q}}\right)$ 处的连续性。 $S=s_{0}$ 表示该点的 弧长值。根据 $\mathrm{G}^{2}$ 连续的定义, $C_{\boldsymbol{P}}(s)$ 关于 $s=s_{0}$ 的一 阶、二阶导数均是连续的。下面验证 $C_{\boldsymbol{Q}}(s)$ 关于 $s=s_{0}$ 的导数的连续性。

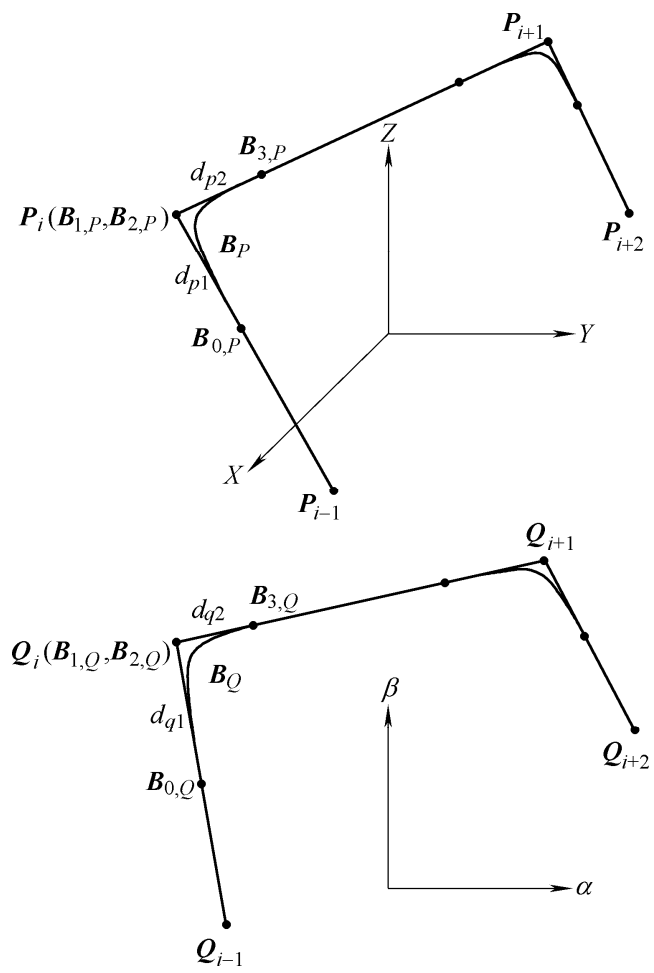

图 3 同步参数化示意图

(1) 关于 $s$ 的一阶导数。

假设 $\left.C_{Q}^{\prime}\right|_{s=s_{0}}$ 表示未转接段在 $s=s_{0}$ 处的一阶导数; $\left.C_{Q}^{\prime}\right|_{s=s_{0}^{+}}$表示过渡曲线在 $s=s_{0}$ 处的一阶导数, 那么

$$
\begin{aligned}
& \left.C_{\boldsymbol{Q}}^{\prime}\right|_{s=s_{0}^{-}}=\frac{\boldsymbol{Q}_{i}-\boldsymbol{Q}_{i-1}}{\left\|\boldsymbol{P}_{i}-\boldsymbol{P}_{i-1}\right\|}=\frac{\left\|\boldsymbol{Q}_{i}-\boldsymbol{Q}_{i-1}\right\|}{\left\|\boldsymbol{P}_{i}-\boldsymbol{P}_{i-1}\right\|} \boldsymbol{T}_{1, \boldsymbol{Q}} \\
& \left.C_{\boldsymbol{Q}}^{\prime}\right|_{s=s_{0}^{+}}=\frac{\mathrm{d} S_{\boldsymbol{Q}}}{\mathrm{d} s}=\left.\frac{3 d_{q 1}(1-t)^{2} \boldsymbol{T}_{1, \boldsymbol{Q}}}{\left\|3 d_{p 1}(1-t)^{2} \boldsymbol{T}_{1, \boldsymbol{P}}\right\|}\right|_{t=0}=\frac{d_{q 1}}{d_{p 1}} \boldsymbol{T}_{1, \boldsymbol{Q}}
\end{aligned}
$$

式中, $T_{1, Q}$ 是线段 $\boldsymbol{Q}_{i-1} \boldsymbol{Q}_{i}$ 的单位矢量。结合(16), 有

$$
\left.C_{\boldsymbol{Q}}^{\prime}\right|_{s=s_{0}^{+}}=\frac{\left\|\boldsymbol{Q}_{i}-\boldsymbol{Q}_{i-1}\right\|}{\left\|\boldsymbol{P}_{i}-\boldsymbol{P}_{i-1}\right\|} \boldsymbol{T}_{1, \underline{Q}}=\left.C_{\boldsymbol{Q}}^{\prime}\right|_{s=s_{0}^{-}}
$$

因此 $C_{\boldsymbol{Q}}(s)$ 在 $s=s_{0}$ 的一阶导数是连续的。

(2) 关于 $s$ 的二阶导数。

设 $\left.C_{\boldsymbol{Q}}^{\prime \prime}\right|_{s=s_{0}^{-}}$表示未转接段在 $s=s_{0}$ 处的二阶导数; $\left.C_{Q}^{\prime}\right|_{s=s_{0}^{+}}$表示过渡曲线在 $s=s_{0}$ 处的二阶导数, 则

$$
\begin{gathered}
\left.\boldsymbol{C}_{\boldsymbol{Q}}^{\prime \prime}\right|_{s=s_{0}^{-}}=\boldsymbol{0} \\
\left.\boldsymbol{C}_{\boldsymbol{Q}}^{\prime \prime}\right|_{s=s_{0}^{+}}=\frac{\mathrm{d}^{2} \boldsymbol{B}_{\boldsymbol{Q}}}{\mathrm{d} s^{2}}=\frac{\mathrm{d}^{2} \boldsymbol{B}_{\boldsymbol{Q}}}{\mathrm{d} t^{2}}\left(\frac{\mathrm{d} t}{\mathrm{~d} s}\right)^{2}+\frac{\mathrm{d} \boldsymbol{B}_{\boldsymbol{Q}}}{\mathrm{d} t} \times\left.\frac{\mathrm{d}^{2} t}{\mathrm{ds}^{2}}\right|_{t=0}
\end{gathered}
$$

根据 Bézier 曲线的表达式，可以得到

$$
\begin{aligned}
& \left.\frac{\mathrm{d}^{2} \boldsymbol{B}_{\boldsymbol{Q}}}{\mathrm{d} t^{2}}\right|_{t=0}=-6 d_{q 1} \boldsymbol{T}_{1, \boldsymbol{Q}} \\
& \left.\frac{\mathrm{d} t}{\mathrm{~d} s}\right|_{t=0}=\frac{1}{3 d_{p 1}} \\
& \left.\frac{\mathrm{d} \boldsymbol{B}_{\boldsymbol{Q}}}{\mathrm{d} t}\right|_{t=0}=3 d_{q 1} \boldsymbol{T}_{1, \boldsymbol{Q}} \\
& \left.\frac{\mathrm{d}^{2} t}{\left.\mathrm{ds}\right|^{2}}\right|_{t=0}=-\frac{\operatorname{dot}\left(\boldsymbol{B}_{P}^{\prime}, \boldsymbol{B}_{P}^{\prime \prime}\right)}{\left\|\boldsymbol{B}_{P}^{\prime}\right\|^{4}}=\frac{2}{9 d_{p 1}^{2}}
\end{aligned}
$$

将式(21)代入式(20), 得到

$$
\left.\boldsymbol{C}_{\boldsymbol{Q}}^{\prime \prime}\right|_{s=s_{0}^{+}}=\boldsymbol{0}=\left.\boldsymbol{C}_{\boldsymbol{Q}}^{\prime \prime}\right|_{s=s_{0}^{-}}
$$

因此 $\boldsymbol{C}_{\boldsymbol{Q}}(s)$ 在 $s=s_{0}$ 的二阶导数连续。

同理, 可证明位于 $\boldsymbol{P}_{i} \boldsymbol{P}_{i+1}$ 与 $\boldsymbol{Q}_{i} \boldsymbol{Q}_{i+1}$ 之间转接点 $\left(\boldsymbol{B}_{3, P}, \boldsymbol{B}_{3, \boldsymbol{Q}}\right)$ 的一阶和二阶导数也连续。

为保证 2 相邻的转接长度之和不超过原路径长 度, $m_{1}$ 和 $m_{2}$ 分别为

$$
\begin{aligned}
& m_{1}=\min \left(\frac{d_{p 1}}{\left\|\boldsymbol{P}_{i}-\boldsymbol{P}_{i-1}\right\|}, \frac{d_{q 1}}{\left\|\boldsymbol{Q}_{i}-\boldsymbol{Q}_{i-1}\right\|}, 0.5\right) \\
& m_{2}=\min \left(\frac{d_{p 2}}{\left\|\boldsymbol{P}_{i+1}-\boldsymbol{P}_{i}\right\|}, \frac{d_{q 2}}{\left\|\boldsymbol{Q}_{i+1}-\boldsymbol{Q}_{i}\right\|}, 0.5\right)
\end{aligned}
$$

$\left(\boldsymbol{P}_{i}, \boldsymbol{Q}_{i}\right)$ 处的转接长度为

$$
\begin{aligned}
& d_{p 1}=m_{1}\left\|\boldsymbol{P}_{i}-\boldsymbol{P}_{i-1}\right\| \quad d_{q 1}=m_{1}\left\|\boldsymbol{Q}_{i}-\boldsymbol{Q}_{i-1}\right\| \\
& d_{p 2}=m_{2}\left\|\boldsymbol{P}_{i+1}-\boldsymbol{P}_{i}\right\| \quad d_{q 1}=m_{2}\left\|\boldsymbol{Q}_{i+1}-\boldsymbol{Q}_{i}\right\|
\end{aligned}
$$

值得注意的是, 式(16)的存在会造成刀尖点和 刀轴方向的光顺曲线不可能同时对称。为了方便接 下来的轨迹插补计算, 将刀尖点的转接曲线设置为 对称 $d_{p}=\min \left(d_{p 1}, d_{p 2}\right)$

$$
d_{q 1}=\frac{\left\|\boldsymbol{Q}_{i}-\boldsymbol{Q}_{i-1}\right\|}{\left\|\boldsymbol{P}_{i}-\boldsymbol{P}_{i-1}\right\|} d_{p} \quad d_{q 2}=\frac{\left\|\boldsymbol{Q}_{i+1}-\boldsymbol{Q}_{i}\right\|}{\left\|\boldsymbol{P}_{i+1}-\boldsymbol{P}_{i}\right\|} d_{p}
$$

设两条线段上的过渡距离分别为 $d_{q 1}=\left\|\boldsymbol{B}_{0} \boldsymbol{Q}_{1}\right\|$ 、 $d_{q 2}=\left\|B_{3} Q_{1}\right\|$, 得到 $\boldsymbol{B}_{\boldsymbol{Q}}(t)$, 如图 4 所示。同时设两条 线段上的过渡距离相等且 $d_{e}=\max \left(d_{q 1}, d_{q 2}\right)$, 得到 $\boldsymbol{B}_{\boldsymbol{Q}, e}(t)$, 如果线性轨迹 $\boldsymbol{B}_{0}-\boldsymbol{Q}_{1}-\boldsymbol{B}_{3}$ 与 $\boldsymbol{B}_{\boldsymbol{Q}}(t)$ 的最大偏 差是 $\varepsilon, \boldsymbol{B}_{0, e}-\boldsymbol{Q}_{1}-\boldsymbol{B}_{3, e}$ 和 $\boldsymbol{B}_{Q, e}(t)$ 的最大偏差是 $\varepsilon_{e}$, 根据文献[9]

$$
\varepsilon \leqslant \varepsilon_{e}
$$

由式 (25) 计算出的 $d_{q 1}$ 和 $d_{q 2}$ 小于等于 $4 \varepsilon_{q} \csc (0.5 \theta)$, 因此刀轴的转接误差可严格满足。

\section{2 转接光顺的轨迹生成}

\section{1 前瞻速度规划}

光顺之后的路径是直线和曲线组成的曲线。曲 


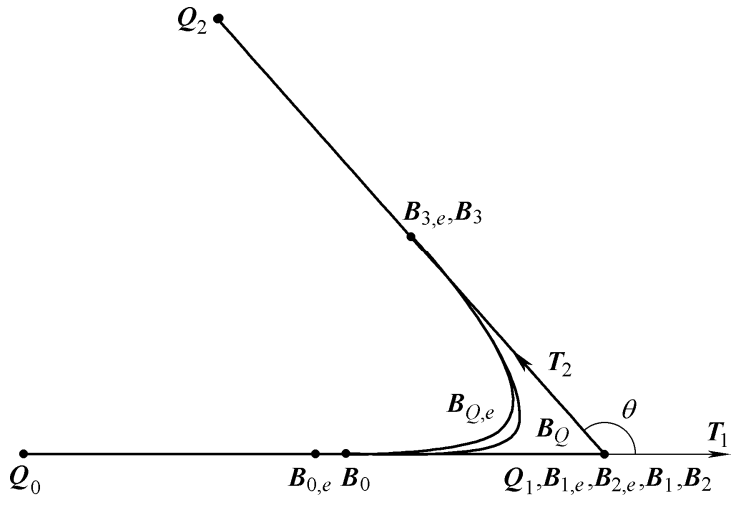

图 4 对称与非对称 Bezier 转接曲线

线的曲率是制约进给速度的重要因素, 曲率越大, 向心加速度越大, 弓高误差越大。因此曲率最大值 点的速度需要进行限制。文献[9]给出, 当 Bézier 曲 线对称, 曲线的曲率极值在曲线中点 $t=0.5$ 处, 且 最大曲率为

$$
\kappa_{\max }=\kappa_{t=0.5}=\frac{4 \sin \theta}{3 d \cos ^{3}(\theta / 2)}
$$

依据光顺后路径的曲率分布情况划分速度规划 单元, 将每个刀位点转接曲线的中点作为分割点, 相 邻 2 个分割点之间的路径进行速度规划和插补计算。

经过转接光顺, 刀具路径实现高阶几何连续性, 但并不意味着平滑的运动。路径的连续性由自身的 几何性质决定。运动的平滑性则是通过位移对时间 的导数来体现, 导数连续性越高, 则运动的平滑性 越好。此外, 限于电机的机械特性, 各伺服轴的速 度, 加速度也需要限制。7 段式 $\mathrm{S}$ 型速度规划方法 以分段函数进行加减速, 可实时规划出跃度有限、 加速度连续的进给速度曲线。相比于 5 段式 $S$ 型速 度规划, 7 段式 $\mathrm{S}$ 型速度规划加速时间更长, 可获 得更高的进给速度。

下面给出基于转接光顺的轨迹生成算法。如 图 5 所示。当对 $\boldsymbol{P}_{i} \boldsymbol{P}_{i+1}$ 进行插补计算时, CNC 系统 预读从 $\boldsymbol{P}_{i}$ 到 $\boldsymbol{P}_{i+m}$ 之间的 $m \geqslant 2$ 条路径, 然后进行以 下步骤。

(1) 路径光顺, 对 $\boldsymbol{P}_{i}$ 和 $\boldsymbol{P}_{i+m-1}$ 之间的路径进行转 接光顺，计算出线段和 Bézier 曲线的表达式。窗口 末端不需要进行光顺, 保证末端执行器能够平稳地 停在 $\boldsymbol{P}_{i+m}$ 。

（2）速度极值计算, 确定 $\boldsymbol{P}_{i+1}^{\prime}$ 点处的速度大小, $\boldsymbol{P}_{i+1}^{\prime}$ 是 $\boldsymbol{P}_{i+1}$ 处光顺曲线的中点。

(3) 速度规划, 根据路径起点和终点的速度、 路径长度、进给率、加速度、跃度, 用 7 段式 $\mathrm{S}$ 型 速度规划得到加速度连续的速度曲线。

(4) 轨迹插补计算, 根据生成的速度曲线对路 径进行插补计算。
光顺方法在第 1 节已经详细介绍。 7 段式 S 型 速度规划方法根据路径长度以及首末两点的速度大 小, 实时规划出加速度连续的进给速度曲线, 具体 细节可参考文献[15]。路径的插补计算可参考文献 [5]。下面主要介绍如何确定终点的速度极值。

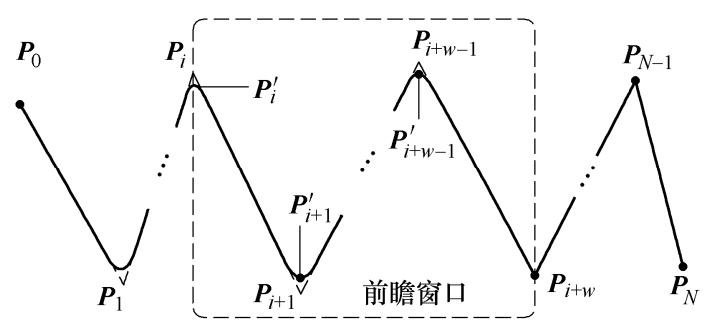

图 5 前瞻速度规划

\section{2 终点的速度极值}

当采用七段式 $S$ 型速度规划时, 需要确定起始 点和终点的速度值。对于每一段路径 $\boldsymbol{P}_{i} \boldsymbol{P}_{i+1}, \boldsymbol{P}_{i}^{\prime}$ 和 $\boldsymbol{P}_{i+1}^{\prime}$ 之间的路径是刀具实际运动的路径。起点 $\boldsymbol{P}_{i}^{\prime}$ 的 速度是上一段的结束速度, 因此只需要确定终点 $\boldsymbol{P}_{i+1}^{\prime}$ 的速度。 $\boldsymbol{P}_{i+1}^{\prime}$ 的速度主要考虑以下 2 方面。

\subsection{1 前向速度极值扫描}

从 $\boldsymbol{P}_{i}^{\prime}$ 按照图 6 加速到 $\boldsymbol{P}_{i+1}^{\prime}$, 可得到考虑加速距 离 $L_{i}$ 、最大加速度 $A_{\text {max }}$ 和最大跃度 $J_{\text {max }}$ 等约束的速 度极值。令起点和终点的速度分别为 $v_{0}$ 和 $v_{e}$, 则存 在如下关系

$$
v_{e}=0.5\left(\sqrt{\frac{A_{\max }^{4}}{J_{\text {max }}^{2}}-4\left(\frac{A_{\max }^{2}}{J_{\max }} v_{0}-v_{0}^{2}-2 L_{i} A_{\max }\right)}-\frac{A_{\max }^{2}}{J_{\max }}\right)
$$

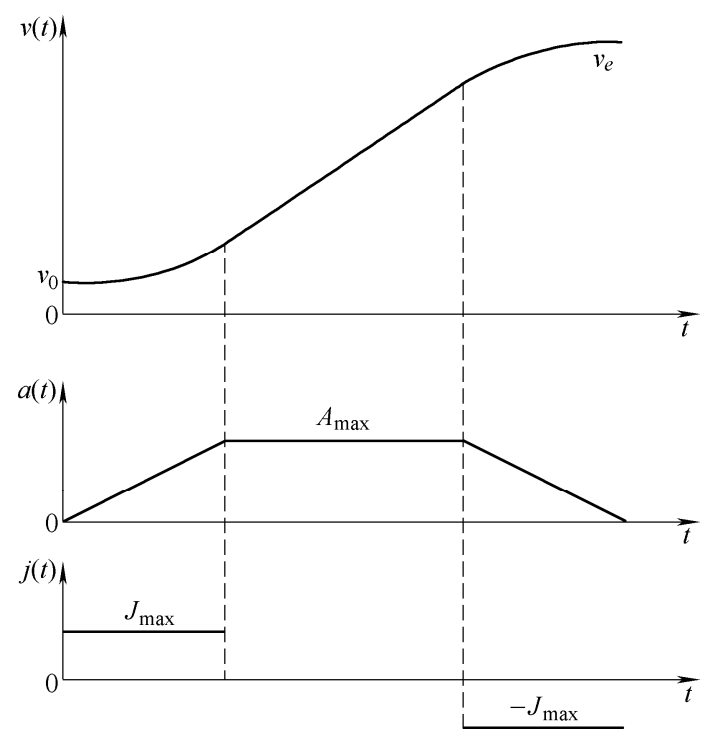

图 6 跃度有限的 $\mathrm{S}$ 型加速

考虑曲率的限制, $\boldsymbol{P}_{i+1}^{\prime}$ 的极值速度

$$
v_{a}=\sqrt{\frac{A_{\max }}{\kappa_{\max }}}
$$

考虑弓高误差 $\delta$ 和插补周期 $T_{s}$ 的限制, $\boldsymbol{P}_{i+1}^{\prime}$ 的 
极值速度

$$
v_{\delta}=\frac{2}{T_{s}} \sqrt{\frac{2 \delta}{\kappa_{\max }}-\delta^{2}}
$$

则 $\boldsymbol{P}_{\boldsymbol{i}+\boldsymbol{1}}^{\prime}$ 处的前向速度极值为

$$
v_{f}=\min \left(v_{e}, v_{a}, v_{\delta}, F\right)
$$

式中, $F$ 是 CAM 刀路指定的最大进给速度。

下面给出式(28)和式(29)的证明。

首先, 证明式(28)。对于只有加速过程的 S 型 速度规划, 根据文献[15], 加速时间

$$
T_{a}=\frac{2 L_{i}}{v_{0}+v_{e}}
$$

加速结束时的速度

$$
v_{e}=v_{0}+\left(T_{a}-T_{j}\right) A_{\max }
$$

其中, $T_{j}$ 是加速度达到最大值的时间

$$
T_{j}=\frac{A_{\max }}{J_{\max }}
$$

结合式(32)、(33)和(34), 可以得到

$$
v_{e}=v_{0}+\left(\frac{2 L_{i}}{v_{e}+v_{0}}-\frac{A_{\max }}{J_{\max }}\right) A_{\max }
$$

整理得到

$$
v_{e}^{2}+\frac{A_{\max }^{2}}{J_{\max }} v_{e}+\left(\frac{A_{\max }^{2}}{J_{\max }} v_{0}-v_{0}^{2}-2 L_{i} A_{\max }\right)=0
$$

式(36)是关于 $v_{e}$ 的一元二次方程, 求解即可得 到式(28)。

当路径的长度 $L_{i}$ 低于临界值

$$
l=2 v_{0}\left(\frac{A_{\max }}{J_{\max }}\right)+J_{\max }\left(\frac{A_{\max }}{J_{\max }}\right)^{3}
$$

将没有匀加速段, $v_{e}$ 不能用式(28)计算。此时 $v_{e}$ 速 度极值的确定请参考文献[5]。

接下来给出式(29)的证明。

证明：曲线上一点的加速度可以如下表示

$$
\boldsymbol{a}=a \boldsymbol{\tau}+\frac{v^{2}}{\rho} \boldsymbol{n}
$$

式中, $v$ 是速度的值, $\tau$ 是曲线的单位切矢量; $a=v^{\prime}$ 是切向加速度的值, $\boldsymbol{n}$ 是曲线的单位法矢量, $\rho=1 / \kappa$ 是该点处的曲率半径。

根据 S 型速度规划的运动规律, $\boldsymbol{P}_{i+1}^{\prime}$ 处的切向 加速度值 $a=0$ 。由于 $\boldsymbol{P}_{i+1}^{\prime}$ 是转接曲线的中点, 曲率 半径 $\rho=1 / \kappa_{\text {max }}$ 。设 $\boldsymbol{P}_{i+1}^{\prime}$ 处的速度值为 $v_{a}$, 当加速度 值为 $\|\boldsymbol{a}\|=A_{\max }$,

$$
\|\boldsymbol{a}\|=\left\|0 \boldsymbol{\tau}+\frac{v_{a}^{2}}{\rho} \boldsymbol{n}\right\|=\kappa_{\max } v_{a}^{2}=A_{\max }
$$

整理可得式(29)。

\subsection{2 反向速度极值扫描}

反向速度极值扫描主要是为了保证能够从 $\boldsymbol{P}_{i+1}^{\prime}$ 平稳减速停在窗口末端 $\boldsymbol{P}_{i+m}$ 。减速运动可以看成反 向加速运动, 因此从窗口未端, 在每一段路径 $\left(\boldsymbol{P}_{i+m} \boldsymbol{P}_{i+m-1}^{\prime}, \cdots, \boldsymbol{P}_{i+2}^{\prime} \boldsymbol{P}_{i+1}^{\prime}\right)$, 从路径的终点到路径的起 点执行 $\mathrm{S}$ 型加速, 计算出末端的速度极值, 并循环迭 代直至得到 $\boldsymbol{P}_{i+1}^{\prime}$ 处的速度极值。具体的执行过程如下。

(1) 令 $j=i+m, v_{r}=0, j \Leftarrow j-1$;

(2) 如果 $j=i+1$, 则结束规划, 否则执行: 从 $\boldsymbol{P}_{j}^{\prime}$ 到 $\boldsymbol{P}_{j-1}^{\prime}$ 进行加速, 令 $v_{0}=v_{r}$ 利用式(31)计算出 $\boldsymbol{P}_{j}^{\prime}$ 的速 度极值 $v_{r}$ 。速度规划方法是在工件坐标系下进行的。 工件坐标系到机床坐标系是非线性映射关系。令 $\boldsymbol{q}$ 表示各轴的参考命令。以弧长参数 $s$ 进行插补计算, 可得到各轴的速度与加速度

$$
\begin{aligned}
& \dot{\boldsymbol{q}}=\boldsymbol{q}_{\mathrm{s}} \dot{\mathrm{s}} \\
& \ddot{\boldsymbol{q}}=\boldsymbol{q}_{\mathrm{s} \mathrm{s}} \dot{\mathrm{s}}^{2}+\boldsymbol{q}_{\mathrm{s}} \ddot{\mathrm{s}}
\end{aligned}
$$

式中, $\boldsymbol{q}_{s}$ 和 $\boldsymbol{q}_{\mathrm{ss}}$ 是 $\boldsymbol{q}$ 关于 $s$ 的一阶、二阶导数, 与刀 具相对于机床的位置有关。从式(40)可以看出, 各 伺服轴的运动不仅与刀具的运动有关, 同时也受非 线性运动学的影响。因此需要验证 $v_{r}$ 是否满足各轴 的速度约束 $\dot{\boldsymbol{q}}_{\text {max }}$ 和加速度约束 $\ddot{\boldsymbol{q}}_{\text {max }}$ 。如果某些轴的 速度或者加速度超出了最大值, $v_{r}$ 须修正为 $v_{r}=\min \left(\dot{\boldsymbol{q}}_{\max } /\left|\boldsymbol{q}_{\mathrm{s}}\right|, \sqrt{\ddot{\boldsymbol{q}}_{\max } /\left|\boldsymbol{q}_{\mathrm{ss}}\right|}\right), j \Leftarrow j-1 。$

最终, $\boldsymbol{P}_{i+1}^{\prime}$ 的速度为

$$
v_{P_{i+1}^{\prime}}=\min \left(v_{f}, v_{r}\right)
$$

\section{3 仿真与试验验证}

\section{1 仿真分析}

为了验证提出的光顺方法和轨迹生成算法, 首 先以文献[7]的路径进行仿真计算。路径信息如所图 7 示。最大进给速度 $F$ 为 $100 \mathrm{~mm} / \mathrm{s}$ 。刀尖点和刀轴的 转接误差分别是 $0.1 \mathrm{~mm}$ 和 $0.1^{\circ}$ 。在前瞻速度规划 规则中, 最大切向加速度 $A_{\text {max }}$ 、最大切向跃度 $J_{\text {max }}$ 设 置为 $250 \mathrm{~mm} / \mathrm{s}^{2}, 5000 \mathrm{~mm} / \mathrm{s}^{3}$, 插补周期 $T_{\mathrm{s}}$ 设定为 0.4 $\mathrm{ms}$ 。机床的结构如图 8 所示。其逆运动学变换为

$$
X=-p_{x} \cos (C)+p_{y} \sin (C)
$$

$$
\begin{gathered}
Y=-p_{x} \cos (A) \sin (C)-y \cos (A) \cos (C)+\left(p_{z}+L_{a c, z}\right) \sin (A) \\
Z=p_{x} \sin (A) \sin (C)+y \sin (A) \cos (C)+\left(p_{z}+L_{a c, z}\right) \cos (A) \\
A=\arccos \left(o_{k}\right) \\
C=\arctan \left(o_{i} / o_{j}\right)
\end{gathered}
$$

式中, $L_{a c, z}=42$ 是机床的偏置参数。机床平动轴 $X, Y, Z$ 的最大速度和最大加速度分别为 $200 \mathrm{~mm} / \mathrm{s}$, $1000 \mathrm{~mm} / \mathrm{s}^{2}$, 旋转轴 $A, C$ 的最大速度和加速度分别 
为 $1 \mathrm{rad} / \mathrm{s}, 10 \mathrm{rad} / \mathrm{s}^{2}$ 。

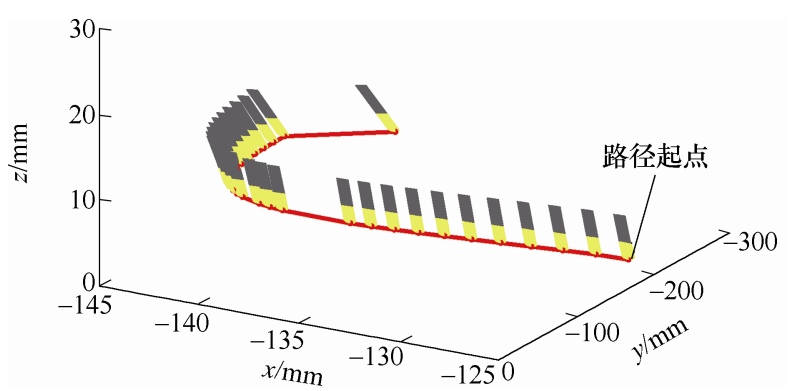

图 7 用于仿真验证的 5 轴刀路

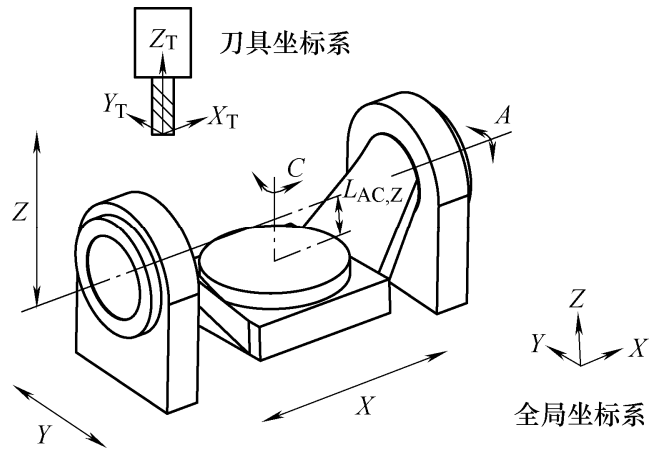

图 8 双回转台 5 轴机床

上述仿真计算在 Matlab 环境下计算。计算机配 置为: Pentium IV $2.4 \mathrm{GHz}$ CPU 和 2 GB RAM。为 了提高进给率，同时避免大的计算量，在前瞻时预 读 4 条线性路径。仿真结果如图 9 12 所示。

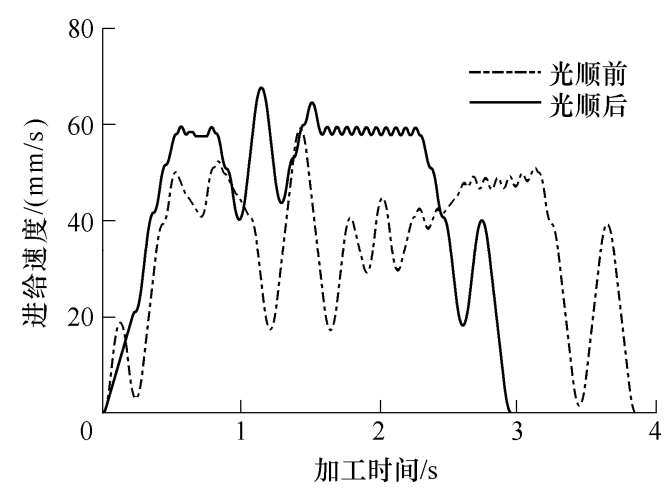

图 9 光顺前与光顺后的进给速度曲线
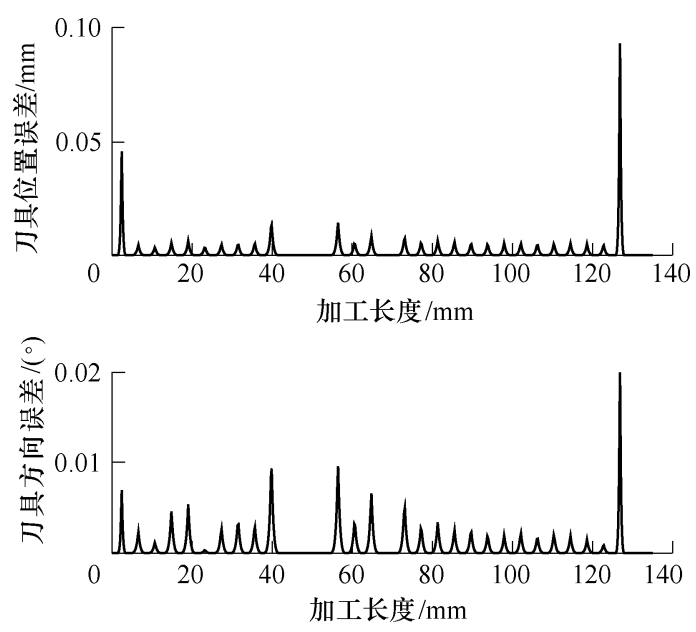

图 10 光顺误差
从图 9 可以看出, 对线性路径进行速度规划时, 加工时间为 $3.85 \mathrm{~s}$; 而光顺之后的加工时间为 $2.95 \mathrm{~s}$, 与 Mori Seiki NMV5000 数控系统预测值 $3.50 \mathrm{~s}$ 相 比, 缩短 $0.55 \mathrm{~s}^{[7]}$ 。各刀位点的距离非常近, 因此无 法加速到最大速度。由于位置和方向在每个离散刀 位点处分别插入了一条 Bézier 曲线, 所以光顺之后 的路径相对于原始路径会存在误差。从图 10 可以看 出刀尖点的最大误差约为 $0.1 \mathrm{~mm}$, 刀轴方向的最大 误差约为 $0.02^{\circ}$, 均没有超过给定值。因此本文采 用的转接方法可以满足转接误差的要求。在确定速 度极值时, 仅考虑速度和加速度等运动学约束, $X$ 轴的速度在局部区域超过最大值, 如图 11 所示; $X 、 Y 、 C$ 轴的加速度在局部区域也超过最大值, 如图 12 所示。当综合考虑运动学和机床运动学特 性, 这些轴的速度和加速度均在限制最大值范围 内, 如图 11 和图 12 所示, 验证了速度极值扫描 方法的合理性。

所提出的转接光顺与轨迹生成算法生成 739 个 插补点, 总耗时 $0.211 \mathrm{~s}$, 平均每个插补点的计算时 间 $0.286 \mathrm{~ms}$, 因此实时性可以满足。
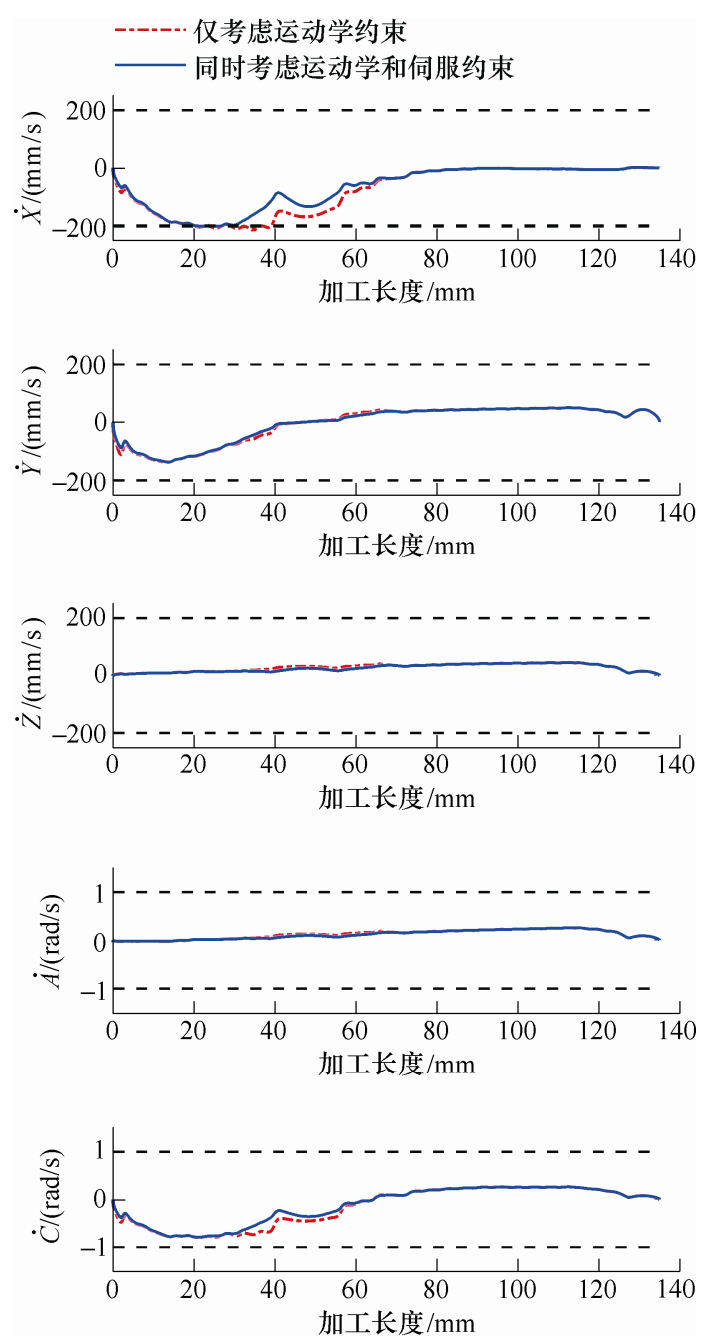

图 11 各轴的速度曲线 

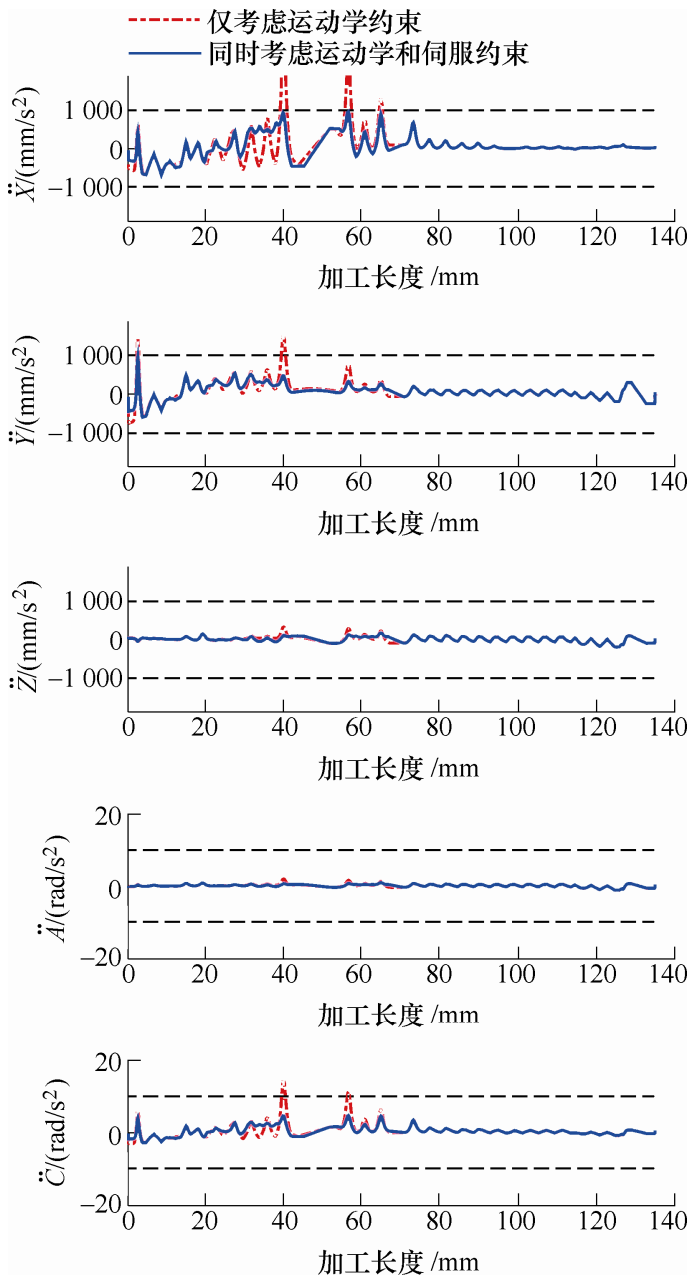

图 12 各轴的加速度曲线

\section{2 试验验证}

针对上面的刀具路径, 接下来在五轴雕铣机上, 验证本文提出的路径光顺和轨迹生成方法。构建试 验平台如图 13 所示, 该试验系统的架构包括以下三 个部分。

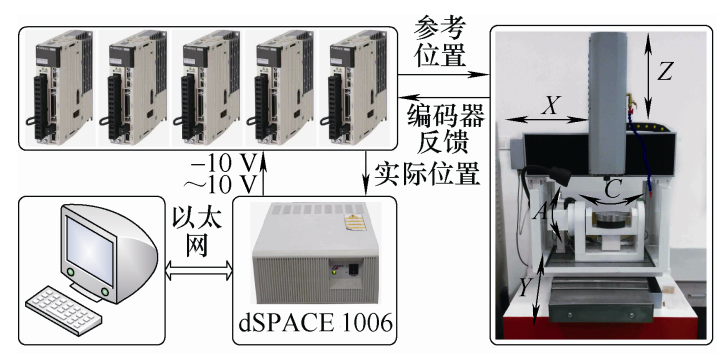

图 13 试验控制平台

(1) 计算机, 配置为 Pentium IV $2.4 \mathrm{GHz}$ CPU 和 2 GB RAM, 用来进行前瞻和插补计算, 最终生 成各轴的参考位置指令, 并利用 Matlab/ Simulink 实现仿真试验的搭建。

(2) dSPACE 实时控制系统, 采用 dSPACE 1006 作为伺服闭环控制器, 用于从计算机主机获取插补 位置指令, 采集各伺服轴光电编码器信号, 并执行
PID 计算和模拟量输出等伺服闭环功能。

(3) 五轴雕铣平台, 各轴采用 YASKAWA SGDV 系列的伺服驱动器和 SGMJV 电机进行驱动, 并采 用丝杜螺母传动, 丝杜导程均为 $10 \mathrm{~mm}$ 。

各轴的跟踪误差如表 1 和图 14 所示。从表 1 和图 14 可以看出, 所提出的路径光顺与轨迹生成算 法有助于提高系统位置控制精度。

\section{表 1 最大跟踪误差}

\begin{tabular}{cccccc}
\hline & $X$ 轴 & $Y$ 轴 & $\begin{array}{c}Z \text { 轴 } \\
/ \mathrm{mm}\end{array}$ & $\begin{array}{c}A \text { 轴 } \\
/ \mathrm{mm}\end{array}$ & $\begin{array}{c}C \text { 轴 } \\
/ \mathrm{mm}\end{array}$ \\
\hline 光顺前 & 0.39 & 0.48 & 0.08 & 0.005 & 0.001 \\
光顺后 & 0.32 & 0.29 & 0.06 & 0.004 & 0.001 \\
\hline
\end{tabular}
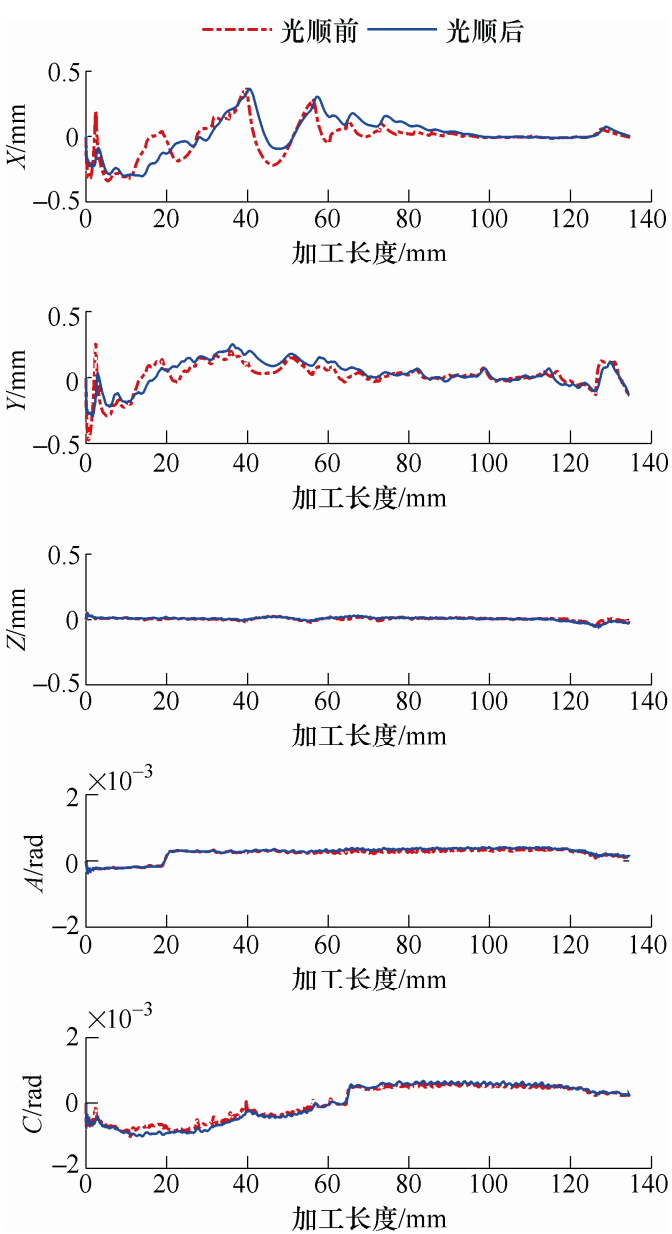

图 14 各轴的跟踪误差

\section{4 结论}

（1）建立了基于欧拉角的路径光顺方法, 满足 G2 连续、误差控制、同步性和实时性要求, 且保证 刀轴矢量单位化。

(2) 所提出的轨迹生成方法结合前瞻技术与 7 段式 S 型速度规划方法, 可以实时获得跃度有限、 加速度连续的速度曲线。相比于 5 段式 $\mathrm{S}$ 型速度, 7 
段式 $\mathrm{S}$ 型速度规划加速时间更长, 可以提高进给率, 缩短加工时间。在速度极值扫描时考虑了非线性运 动学特性, 每段路径的速度均满足伺服约束, 进而 整个路径满足伺服约束。

(3) 仿真与试验表明所提出的路径光顺与轨迹 生成方法可满足误差要求, 提高进给速度, 各驱动 轴的速度和加速度均光滑连续, 没有超过最大值约 束，适合实时计算的环境。

\section{参 考 文 献}

[1] LASEMI A, XUE D, GU P. Recent development in CNC machining of freeform surfaces: A state-of-the-art review [J]. Computer-Aided Design, 2010， 42(7): 641-654.

[2] LIN M T, TSAI M S, YAU H T. Development of a dynamics-based NURBS interpolator with real-time look-ahead algorithm[J]. International Journal of Machine Tools and Manufacture, 2007, 47(15): 2246-2262.

[3] PATELOUP V, DUC E, RAY P. Bspline approximation of circle arc and straight line for pocket machining[J]. Computer-Aided Design, 2010， 42(9): 817-827.

[4] BEUDAERT X, LAVERNHE S, TOURNIER C. Feedrate interpolation with axis jerk constraints on 5-axis NURBS and G1 tool path[J]. International Journal of Machine Tools and Manufacture, 2012, 57: 73-82.

[5] ZHAO Huan, ZHU Limin, DING Han. A real-time look-ahead interpolation methodology with curvaturecontinuous B-spline transition scheme for CNC machining of short line segments[J]. International Journal of Machine Tools and Manufacture, 2013， 65: 88-98.

[6] YUEN A, ZHANG Ke, ALTINTAS Y. Smooth trajectory generation for five-axis machine tools[J]. International Journal of Machine Tools and Manufacture, 2013, 71: 11-19.

[7] LEI W T, SUNG M P, LIN L Y, et al. Fast real-time NURBS path interpolation for CNC machine tools[J]. International Journal of Machine Tools and Manufacture, 2007, 47(10): 1530-1541
[8] LANGERON J M, DUC E, LARTIGUE C, et al. A new format for 5-axis tool path computation, using Bspline curves[J]. Computer-Aided Design, 2004 , 36(12) : 1219-1229.

[9] BI Qingzhen, WANG Y, ZHU Limin, et al. An algorithm to generate compact dual NURBS tool path with equal distance for 5-axis NC machining[M]// Intelligent Robotics and Applications. Springer Berlin Heidelberg, 2010: 553-564.

[10] SHI Jing, BI Qingzhen, ZHU Limin, et al. Corner rounding of linear five-axis tool path by dual PH curves blending[J]. International Journal of Machine Tools and Manufacture, 2015, 88: 223-236.

[11] BEUDAERT X, LAVERNHE S, TOURNIER C. 5-axis local corner rounding of linear tool path discontinuities[J]. International Journal of Machine Tools and Manufacture, 2013, 73: 9-16.

[12] TULSYAN S, ALTINTAS Y. Local toolpath smoothing for five-axis machine tools[J]. International Journal of Machine Tools and Manufacture, 2015, 96: 15-26.

[13] BI Qingzhen, SHI Jing, WANG Yuhan, et al. Analytical curvature-continuous dual-Bézier corner transition for five-axis linear tool path[J]. International Journal of Machine Tools and Manufacture, 2015, 91: 96-108

[14] FARIN G. Curves and surfaces for computer-aided geometric design: a practical guide[M]. Amsterdam: Elsevier, 2014.

[15] BIAGIOTTI L, MELCHIORRI C. Trajectory planning for automatic machines and robots[M]. London: Springer Science \& Business Media, 2008.

作者简介: 赵欢(通信作者), 男, 1983 年出生, 博士, 副教授。主要研 究方向为机器人智能化加工数控装备与技术。

E-mail: huanzhao@hust.edu.cn

张永红, 男, 1991 年出生, 硕士研究生。主要研究方向为数控和运动控 制技术。

E-mail: sczhang@foxmail.com

丁汉, 男, 1963 年出生, 教授, 博士研究生导师。主要研究方向为数字 化制造与智能化制造。

E-mail: dinghan@hust.edu.cn 